\title{
Instalación Leviatán, modelo dual de interacción: Multiusuario y performativa
}

\section{Leviathan installation, dual model of interaction: Multiuser and performative}

\author{
DOI: $10.46814 / 1 a j d v 4 n 1-017$
}

Recebimento dos originais: 01/01/2021

Aceitação para publicação: 28/02/2022

\author{
José Ma Alonso Calero \\ Departamento de Arte y Arquitectura, Facultad de Bellas Artes, Universidad de Málaga \\ Campus El Ejido nº1, Málaga, España \\ E-mail: alo@uma.es
}

\section{RESUMEN}

Leviatán es una plataforma interactiva multimodal de carácter reactivo, una interfaz que se propone como modelo para el estudio de la presencia en entornos de interacción física. La propuesta expone la versatilidad del modelo de interacción sobre las versiones Leviatán 1.0 y Leviatán 2.0. Esta versatilidad se refiere al doble modelo abierto, por un lado, como plataforma interactiva multiusuario (usuarios no entrenados en la plataforma) y por otra como instalación performativa para un solo performer (usuario entrenado en la plataforma). Se presentan en este artículo los detalles técnicos de la implementación en Arduino y Processing para controlar sensores de barrera y sónares, proyecciones de imágenes de síntesis y diodos LED, todo ello para detectar la presencia de los usuarios y dar respuesta a la interacción del usuario por medio de actuadores implementados en el interfaz físico.

Palabras clave: Instalación artística, interacción física, arte reactivo, performance interactivo, multiusuario.

\begin{abstract}
Leviathan is a multimodal interactive platform of reactive character, an interface that is proposed as a model for the study of presence in physical interaction environments. The proposal exposes the versatility of the interaction model on Leviathan 1.0 and Leviathan 2.0 versions. This versatility refers to the double open model, on the one hand, as a multi-user interactive platform (users not trained in the platform) and on the other hand, as a performative installation for a single performer (user trained in the platform). This paper presents the technical details of the implementation in Arduino and Processing to control barrier and sonar sensors, synthesis image projections and LED diodes, all to detect the presence of users and respond to user interaction by means of actuators implemented in the physical interface.
\end{abstract}

Keywords: Installation art, physical interaction, reactive art, interactive performance, multi-user.

\section{INTRODUCCIÓN}

En este artículo se plantea la necesidad de crear modelos basados en la interacción (Interaction driven. Parés, Narcís \& Roc [6]) desde donde investigar el desarrollo de contenidos abiertos que estén a mitad de camino entre la investigación empírica y la creación artística, y desde donde se propone la creación de modelos de interacción. Este ámbito se podría concretar en instalaciones para la 
investigación en presencia por un lado, de forma empírica, y por otro nos daría la posibilidad de generar y producir propuestas de contenido artístico desde un perfil puramente performativo.

Los referentes que tomamos para nuestra investigación se centran en el estudio y en la experimentación sobre modelos de interacción, así como en el desarrollo de modelos genéricos. El objetivo principal que nos planteamos no se centra en qué vemos sino en cómo nos relacionamos con. Tal y como proponen Parés y Parés (2001), centramos nuestra investigación en el mecanismo de interacción, (interaction-driven), y no en el mundo interactivo que se representa (content-driven). [6]

Planteamos que la concepción del espacio forma parte del dispositivo de interacción. Así, el espacio es un referente de nuestro interfaz físico, una metáfora similar o real del entorno sin el hándicap del protocolo, donde el nivel de percepción del usuario determina la experiencia y el alcance o ámbito de interacción con el mundo físico, real o virtual.

Partimos de la idea de segunda piel, en referencia al lema de la 9a edición de ART FUTURA, "La segunda piel”, que es un homenaje a Marshall McLuhan cuando en los años sesenta decía que "en la Era Eléctrica todos llevaremos a la humanidad como nuestra segunda piel". [5]

La idea de esa segunda piel sería la conexión con el mundo post-contemporáneo y postelectrónico inmerso en la conectividad. Esa piel creada artificialmente por el hombre define la forma en que nos relacionamos con el mundo, es como otra capa de cebolla con otro nivel de percepción, esa segunda piel nos coloca en una situación de aislamiento para luego enviarnos de vuelta esa realidad amplificada. Esa segunda piel funciona como filtro, tamiz en ambos sentidos, es nuestro interlocutor físico que da información a la vez que la absorbe.

Se trata de una segunda piel que construye sobre nosotros una realidad ensimismada, cual espejo que refleja un mundo similar a nosotros, es ahí cuando intentamos estructurar esa realidad paralela y nos planteamos la construcción de ese mundo virtual por medio de la tecnología, la cual nos recubre con escafandras tecnológicas y exoesqueletos robotizados. La tecnología se concibe como una extensión del cuerpo, una hibridación que en sentido inverso se aproxima al carácter de implante tecnológico cual obra de Eduardo Kac o de Stelarc. ¿En qué punto podemos colocar esa barrera o límite donde el interfaz separa nuestra percepción de nuestro cuerpo sensorial? Es una frontera indefinida donde conectar con la otra realidad. En este sentido hay diferentes apuestas, una sería la que se nos podría presentar en forma de química artificial, conectada a nivel neuronal; otra podría ser la que se ubicaría directamente enraizada en nuestras extensiones sensoriales para alimentarlas, para acapararlas de una forma muy específica al igual que esos primeros y rudimentarios dispositivos multimodales de realidad virtual como el Sensorama; otra podría formar parte de nuestra escena de visualización donde todo se nos presenta en un modo o lenguaje diferente, icónico, comunicacional; un alfabeto contemporáneo de elementos formados por patrones cual realidad aumentada. 


\subsection{ARTE REACTIVO}

En este punto nos cuestionamos aspectos sobre el modelo de interacción que consideramos más cercano a un modelo llamado "reactivo" más que "interactivo". Esto implica la ausencia de un objetivo, el cual se establecería previamente por medio del protocolo de la experiencia y que llamaremos leit motiv. Con la eliminación del objetivo de la experiencia logramos potenciar la creación por parte del usuario de su propia experiencia, su propio drama.

Esto plantea el problema de la implicación del usuario en la experiencia, pues si no marcamos un leit motiv, ¿Cómo lograremos que el usuario se involucre en la experiencia con la finalidad de conseguir un alto grado de presencia?

Sin un leit motiv predefinido involucramos al usuario mediante algún mecanismo que cumpla la función del hilo de Ariadna. Estos mecanismos podrían ser la complejidad de los comportamientos que forman parte del mundo global. Nos planteamos crear una obra de arte reactivo, sin leit motiv y con un drama interactivo (del lado del usuario) mediante un hilo de Ariadna.

Sobre la tipificación de interacción reactiva, donde el usuario construye su propia experiencia, M. Schultz [7] nos hace este análisis sobre interacción y libertad:

"La interacción real, en Red, se construye sobre la noción de libertad (aunque sea relativa). Si no se acepta la libre intervención, no se puede acceder a la idea de interactividad. Sin la aceptación de la intervención libre, sólo se aportaría combustible para los condicionamientos sociales o al mecanismo de estímulo y respuesta (como lo hicieron Skinner, Watson, Pávlov). El concepto para calificar esos mecanismos es inter-reacción. Expresa un comportamiento solamente reactivo, no proactivo o de iniciativas personales. La presencia de mecanismos entrenados de estímulo y respuesta no resulta coherente con la idea de las bifurcaciones y/o trayectos libremente elegidos por el usuario”.

Para R. Lozano-Hemmer [4] está claro la definición de arte reactivo a partir de las relaciones del público con la obra, dejando del lado del público el peso de la participación en la experiencia:

"El arte reactivo, en su definición más amplia, es aquél que cambia con la presencia y actividad del público. En el arte reactivo el espectador no espera a que la obra se desdoble ante sus ojos, sino al contrario, es la obra la que aguarda a que el espectador haga algo, para entonces reaccionar de una forma u otra. Los ordenadores facilitan esta vocación de vigilancia: las obras ven, escuchan y sienten al público, y se comportan según el mensaje o sensación que cada artista desee programar."

En el caso de C. Gianetti [2], ella pone el peso de la definición que hace de sistemas reactivos en el acceso multidireccional de una postulada interacción, que conlleva una estructuración de los contenidos, al fin y al cabo, predeterminados:

"Injerencia en un programa a través de la estructuración de su desarrollo en el ámbito de las posibilidades dadas. Se trata de una interactividad de selección, que implica la posibilidad de acceso 
multidireccional a informaciones audiovisuales para la ejecución de operaciones predeterminadas por el sistema, y por lo tanto limitadas a éstas".

Y es precisamente sobre el hilo de Ariadna sobre el que R. Lozano-Hemmer [4] hace la siguiente descripción de la obra de arte reactivo Satori (obra de Mario Canali y Marcello Campione)

"No hay ningún objetivo que alcanzar, ningún enemigo al que destruir, ningún invasor que rechazar, ninguna labor que llevar a cabo más que la exploración del espacio, siguiendo el hilo de Ariadna de la curiosidad individual y las emociones, en una personal búsqueda del significado”.

Esto nos sitúa en el planteamiento anteriormente hecho donde el hilo de Ariadna es el mecanismo que engrasa la obra reactiva donde no tenemos un leit motiv predefinido.

\subsection{REFERENTES}

Por condición tecnológica los dispositivos, sensores y actuadores han determinado el carácter de los entornos de inmersión donde su definición no viene o venía dada por una búsqueda en el modelo de interacción, siendo éste el objetivo principal para dar respuestas a las experiencias sensoriales del usuario o espectador. Por ello, creemos que es fundamental centrar el análisis y estudio de esta materia en el modelo de interacción.

Como plataformas referentes, cuyos diseños se basan en modelos similares de interacción, citaremos la plataforma desarrollada por nuestro grupo de investigación, Asteroide B612, Fig.1, [1] plataforma interactiva basada en una proyección cenital de un asteroide, una propuesta de interfaz físico con carácter reactivo.

En relación a la concepción espacial debemos mencionar la propuesta del Panoscope, Fig.2, de Luc Courchesne de la Universidad de Montréal, que plantea un interfaz físico cóncavo, que cubre el espacio de visualización por debajo de la mirada.

Fig.1. Panoscope.

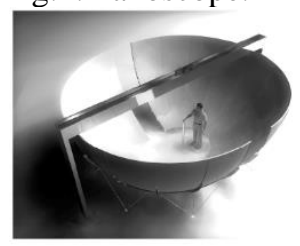

Fig.2. Asteroide B612.

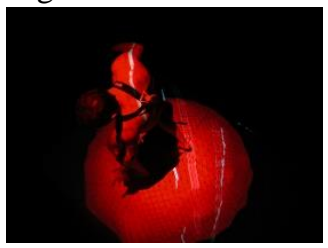

En relación al trabajo con LEDS debemos mencionar el trabajo de interacción con luces LEDS de Takayuki Fujimoto, componente y director de escena de Dump Type, y de relevante interés para nuestra versión 2.0 de Leviatán. Destacar dos de sus proyectos: Refined Color, Fig.3, y True Drawing, Fig.4. El primero es un espectáculo concebido a la italiana donde hay un gran protagonismo del juego de luces con los performers. El segundo es una acción para espacios no jerarquizados donde el público 
está al mismo nivel que el performer, que interactúa con su sombra proyectada alrededor de él por un juego de focos LEDS de colores, la sombra es producida por un anillo de focos situada a una altura de $4 \mathrm{~m}$ que posibilita un rico juego de sombras coloreadas que rotan alrededor del performer.

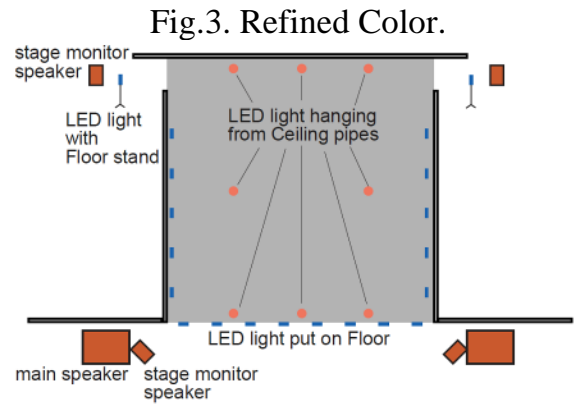

Fig.4. True Drawing.

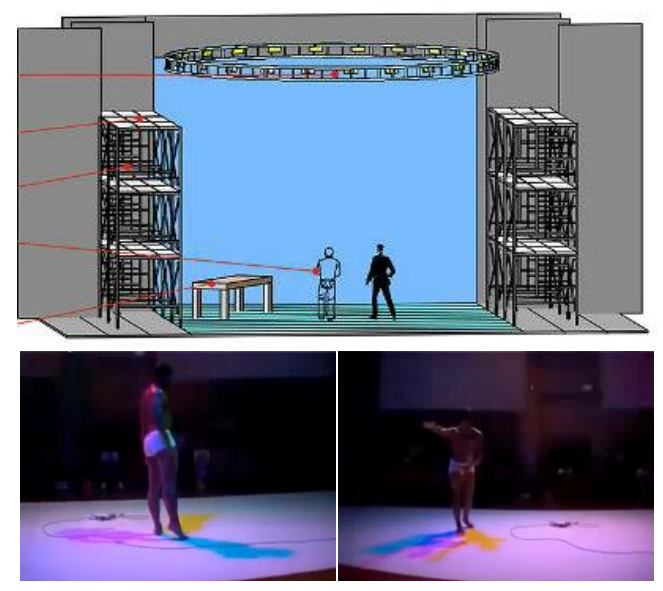

\section{DESCRIPCIÓN DE LA METÁFORA}

Leviatán es una plataforma que forma parte de un proyecto de investigación en interacción hombre-máquina. Esta plataforma es un entorno de inmersión que simula el interior de un ser, una bestia marina, el Leviatán.

Leviatán es un vocablo proveniente del nombre hebreo clásico Livyatan, monstruo bíblico que vivía en el mar. Puede ser traducido como monstruo del mar, cocodrilo o serpiente gigante, considerándose sinónimo de cualquier criatura acuática de gran tamaño. En hebreo moderno, significa simplemente "ballena".

Algunas interpretaciones del Génesis aducen que Dios creó un Leviatán masculino y otro femenino, luego mató a la hembra, la saló y se la ofreció en alimento a los justos, porque si el Leviatán se procreara, el mundo no podría más que someterse ante él.

El Leviatán se puede interpretar también como el mar mismo. Algunos eruditos lo han entendido como una referencia bíblica metafórica de los temibles animales marinos que aterrorizaron el reino de Israel. 
Se tomó este referente como metáfora para la concepción del diseño del interfaz, buscando estar “dentro de", en el interior del mundo virtual, engullido, en el vientre de la bestia, cual Pinocho engullido por la ballena o como el propio Jonás en la Biblia.

\section{DESCRIPCIÓN DE LA PLATAFORMA}

La plataforma interactiva multimodal de carácter reactivo Leviatán ha sido montada en dos experiencias distintas hasta el momento, Leviatán 1.0 y Leviatán 2.0. Cada una de las experiencias proponía un sistema de sensores-actuadores diferente sobre el mismo interfaz físico, una especie de carpa, cuyo objetivo último es el estudio de un modelo abierto de interacción. Ambas experiencias han sido concebidas, desarrolladas e implementadas dentro de los proyectos de investigación en interacción hombre-máquina que se desarrolla dentro del grupo de investigación DIANA (Diseño de Interfaces Avanzados. Grupo de investigación TIC171 del Plan Andaluz de Investigación. PAI).

La plataforma genérica está compuesta por los siguientes elementos comunes:

- La carpa, como el interfaz físico, elemento escénico principal.

- Los dispositivos, sensores y actuadores.

- El modelo de interacción.

- El contenido o generación de la experiencia.

\subsection{LA EXPERIENCIA LEVIATÁN 1.0}

Esta primera experiencia se llevó a cabo con público real en el Art Futura Show 2007 celebrado en El Parque de las Ciencias de Andalucía, del 25 de octubre al 4 de Noviembre 2007 en Granada.

Estos son los dispositivos utilizados:

- Sensores de posición combinados en un solo eje.

- Actuadores en forma de animaciones interactivas en proyección de vídeo.

- Circuito cerrado de TV como retroalimentación, para que desde el exterior de la carpa se vea el comportamiento de los usuarios en el interior.

\subsection{LA EXPERIENCIA LEVIATÁN 2.0}

En esta segunda experiencia, que se describe en detalle en este artículo, se ha hecho un desarrollo técnico más ambicioso, pero sin público real. El desarrollo avanzado implementa una integración de sensores sónares y, cómo actuadores, un sistema de LED combinados para lograr un juego de luces en toda la gama RGB, junto a proyecciones de imagen de síntesis creadas con Processing. 
La estructura del desarrollo está compuesta por un sistema de nodos, conectados por comunicación inalámbrica Zigbee e integrado con Processing y Arduino. Además del desarrollo de los actuadores compuesto por un sistema de LED que generan el juego de color-luz por combinación de la colorimetría RGB.

\section{ESTRUCTURA TÉCNICA DEL PROTOTIPO LEVIATÁN 1.0}

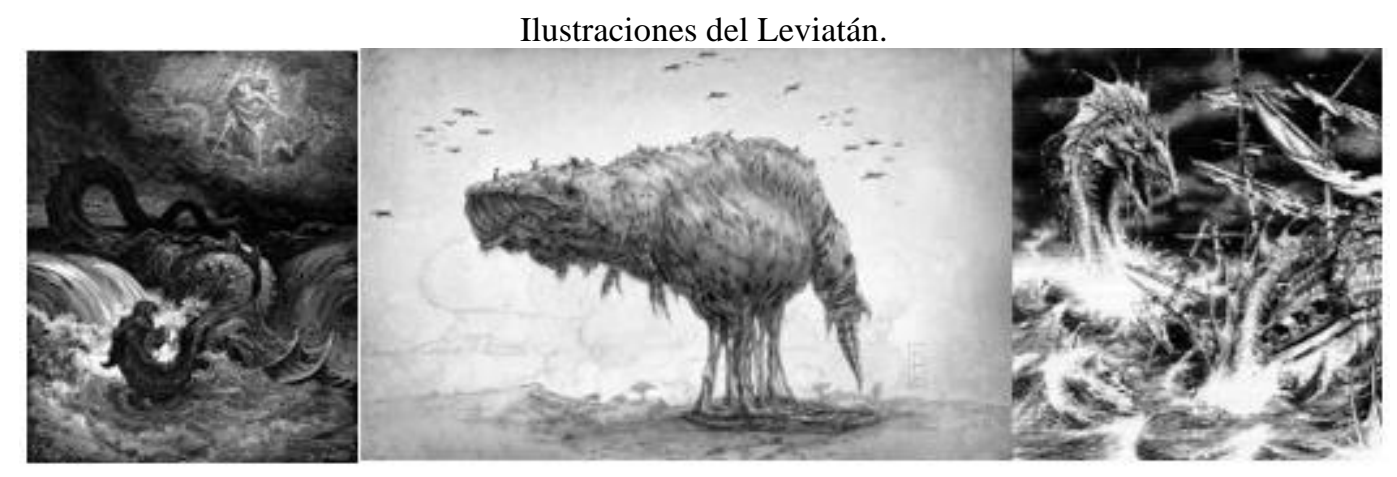

El elemento principal de la instalación es una carpa de $6 \mathrm{~m}$ de largo por $3 \mathrm{~m}$ de ancho y $3 \mathrm{~m}$ de alto. Desde el exterior, sobre la carpa, que es usada como pantalla de proyección, se proyectan una serie de imágenes de síntesis. La proyección sobre la carpa es visible desde fuera por el público, buscando una implicación pasiva, y también desde dentro por los usuarios que interactúan. Estos usuarios se introducen dentro de la carpa por uno de los laterales que se pliega y despliega a modo de capota. Una vez dentro, el usuario se encuentra un espacio que está definido para desplazarse en una sola dirección y en ambos sentidos a lo largo de la carpa. Los usuarios pueden explorar los diferentes elementos interactivos que se proyectan sobre la carpa. Estos eventos suceden en función del movimiento que realizan los usuarios y que son recogidos por los sensores. Un elemento interesante en esta primera versión es la colocación de una cámara de circuito cerrado de TV que muestra la imagen del interior de la carpa a los usuarios antes de entrar, anticipándoles información de cómo funciona. Este dispositivo de retroalimentación actúa como protocolo de introducción al uso de la plataforma. 

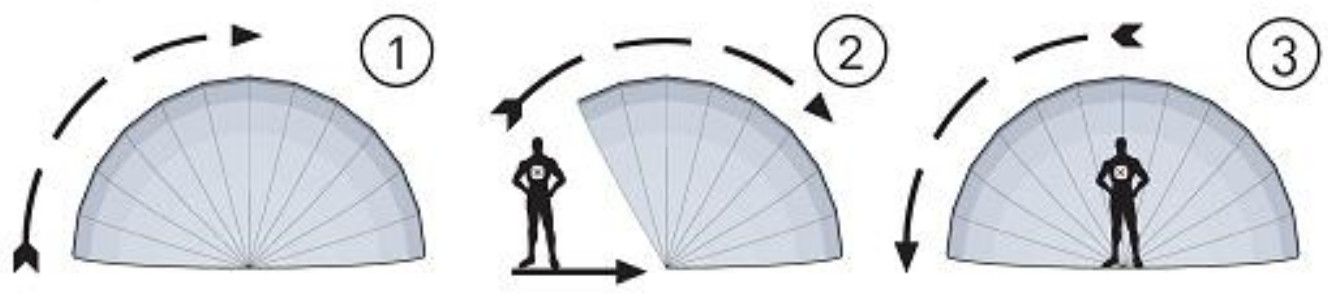

Apertura y entrada a la carpa
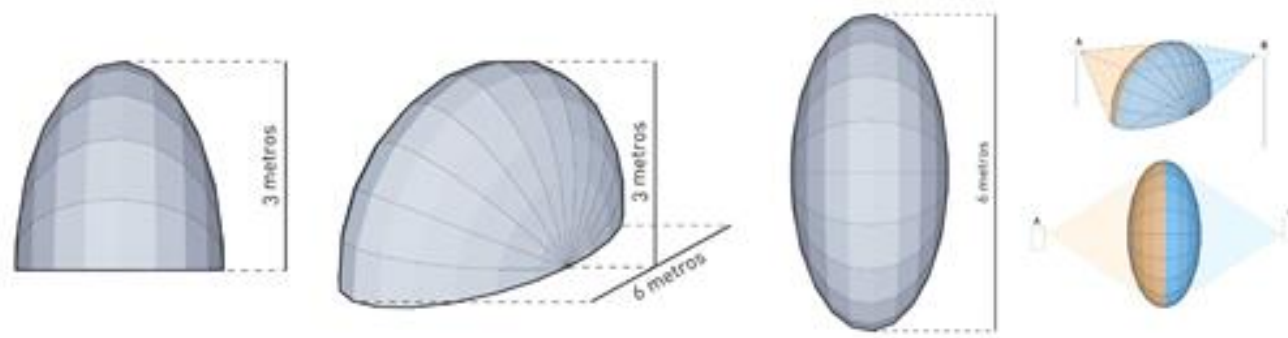

Dimensiones de la carpa y proyecciones laterales.
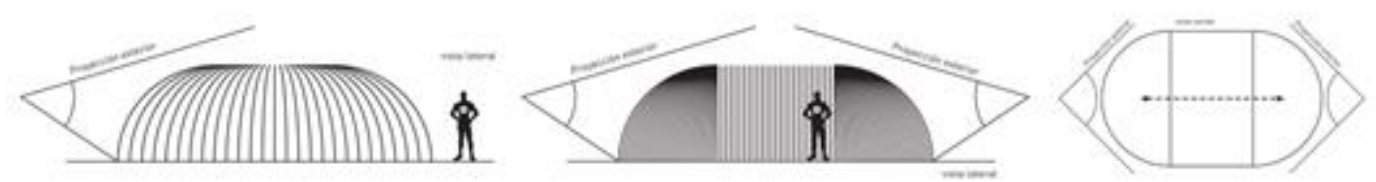

Proyecciones de un extremo a otro de la carpa.

\section{ESTRUCTURA TÉCNICA DEL PROTOTIPO LEVIATÁN 2.0}

\subsection{DESCRIPCIÓN DEL SISTEMA}

La segunda versión de leviatán 2.0 incorpora, respecto a Leviatán 1.0, un sistema que consiste en una red inalámbrica de sensores y actuadores, basados en sonar y focos LED respectivamente. Para el transporte de la información se ha utilizado la tecnología 802.15.4, base sobre la que se define Zigbee que permite una mayor autonomía en cada nodo. Como actuador principal se ha diseñado e implementado un sistema para realizar efectos de luz basados en la tecnología LED. Se ha querido realizar una plataforma versátil para poderse utilizar de forma flexible en otros modelos de interacción. Como soluciones de lenguaje de programación se ha elegido Processing y como herramienta de prototipado rápido, Arduino.

El sistema ha sido diseñado para poder interactuar con el entorno en tiempo real. Hemos procurado utilizar tecnologías que permitan una fácil incorporación de nuevos elementos dejando abierta la posibilidad de una posible realización de Leviatán 3.0.

Nuestro sistema se compone de 4 nodos, que hemos conectado de manera inalámbrica. Nuestra red tiene un nodo principal que gestiona la información que recibe de los demás nodos. Este nodo es 
el interfaz con nuestro programa principal programado en el leguaje Processing que se ejecutará en un ordenador. Tenemos otros dos nodos implementados mediante Arduino que monitoriza la actividad que existe dentro de la carpa mediante un sensor sonar. Tras procesar la información la envía al nodo principal. El programa principal tiene dos tipos de actuadores, las dos visuales. El primero de ellos es un nodo también implementado mediante Arduino al que se le conectan dos focos de diodos LED. La comunicación con este nodo también se realiza de manera inalámbrica.

El otro tipo de actuador visual son dos proyectores que irán proyectando sobre la carpa efectos visuales programados en Processing.

Los módulos sónares realizan una medida cada cierto tiempo. Este tiempo irá variando según las medidas que vaya realizando de manera que si dentro de la carpa no hay nadie, el sistema irá espaciando las muestras para reducir el consumo y prologar de esta manera la vida de la batería. Si las mediciones detectan que hay gente dentro de la carpa la frecuencia aumentará y si las mediciones indican que hay actividad cerca del sensor, las mediciones alcanzarán su máxima frecuencia, ya que consideraremos que la persona está exigiendo al sistema una máxima interacción.

El módulo de diodos LED tiene como función gestionar cada uno de los colores de los diodos para proporcionar una amplia gama de colores dentro de la carpa. Se han programado una serie de funciones básicas que corresponden a diferentes efectos visuales.

El módulo central gestiona toda la información que se genera en la red e incluso realiza animaciones para ser proyectadas en el exterior de la carpa.

\subsection{ARDUINO, PLATAFORMA HARDWARE}

Aunque en el mercado hay un gran número de microcontroladores que se adecuan a nuestras necesidades de manera eficaz, tales como Phidgets o Parallax, Arduino ofrece un precio mucho más competitivo y un entorno denominado open Hardware en el que cada usuario puede extender las funcionalidades tanto de la placa de prototipado como del entorno de programación para adecuarlas a sus necesidades.

Establecimos como requisito inicial que nuestro sistema debe tener una autonomía mínima de 12 horas, ya que sería el tiempo mínimo que debería estar en funcionamiento antes de poder ser recargadas las baterías de las motas. Arduino tiene un consumo muy bajo que nos permite elegir entre un amplio rango de soluciones para implementar el sistema de alimentación.

Podemos configurar las salidas de comunicación de Arduino como fuentes de señal PWM. Además, la frecuencia fundamental de la señal PWM es de 500 Herzios, más que suficiente para gestionar los diodos encargados de los efectos luminosos que describiremos posteriormente. 
Además, Arduino soporta comunicación serie con otros dispositivos. Esta característica nos ha facilitado la comunicación con la antena para la gestión de la red inalámbrica ocupando así un número de pines de comunicación mínimo.

\subsection{COMUNICACIÓN I2C.}

Este protocolo de comunicaciones está muy extendido. Fue ideado para la comunicación de un microcontrolador con periféricos. En nuestro sistema es utilizado para comunicar los sensores con Arduino. Actualmente existe una infinidad de dispositivos que utilizan este protocolo para funcionar por lo que también nos permitirá ampliar la funcionalidad en posibles versiones del Leviatán.

\subsection{DIODOS LED}

Una característica muy importante de los diodos LED es que podemos modelar el espectro de la luz emitida en el proceso de fabricación. De este modo si emitimos una luz con un espectro muy estrecho será similar a emitir una luz de un color definido. Otra de las características en que destacan los diodos LED es que el tiempo de subida de la señal de luz es muy pequeño. Esto permite que un foco de diodos LED pueda ser controlado mediante una señal PWM o proporcionar efectos estroboscópicos. Este hecho nos permitirá controlar la intensidad de iluminación reduciendo o ampliando el ciclo de trabajo de la señal. Esto nos va a permitir realizar una gran cantidad de efectos visuales de manera sencilla y eficaz que hace solo una década hubiera resultado imposible.

Para poder utilizar Arduino como fuente de la señal PWM hemos tenido que diseñar e implementar por completo el sistema de iluminación. Este circuito consta de una fuente de alimentación de tensión regulable que alimenta las baterías de diodos. El control de la iluminación se ha realizado mediante transistores MOSFET de potencia ya que trabajamos con magnitudes de corriente elevadas. 


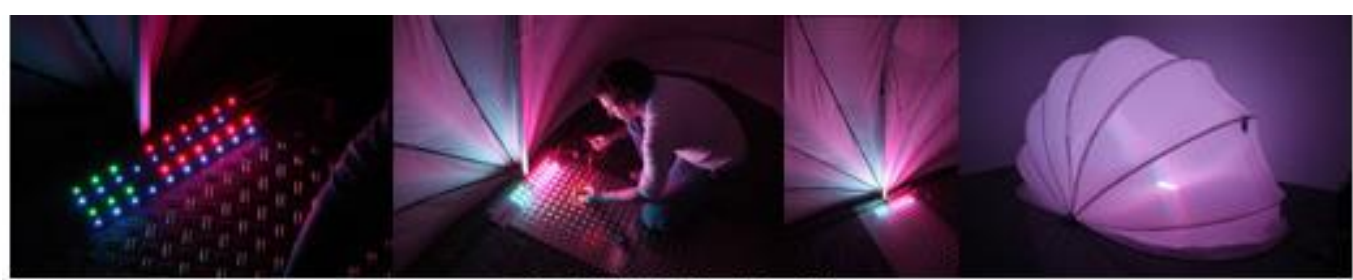

Instalación de Leviatán 2.0

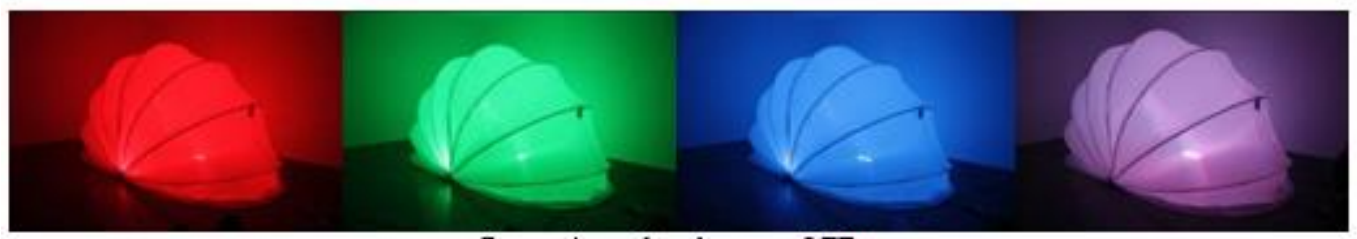

Proyecciones de colores con I.EDz.

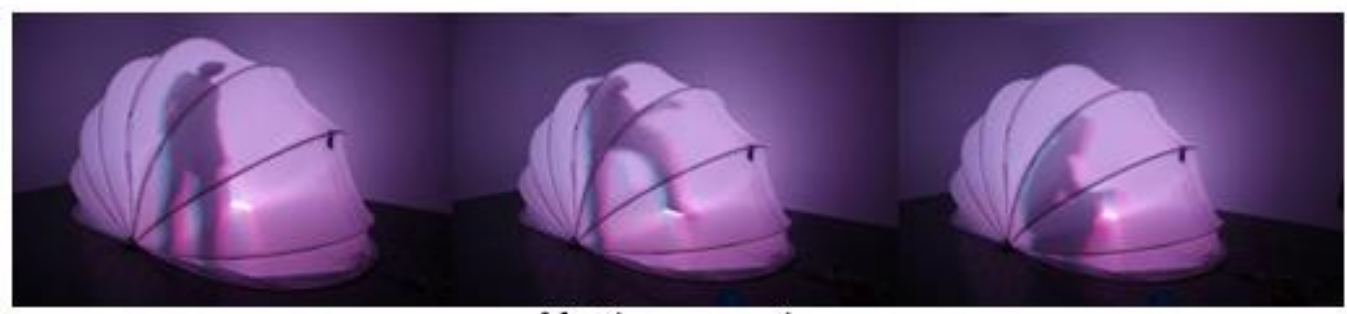

Montaje con un usuario.

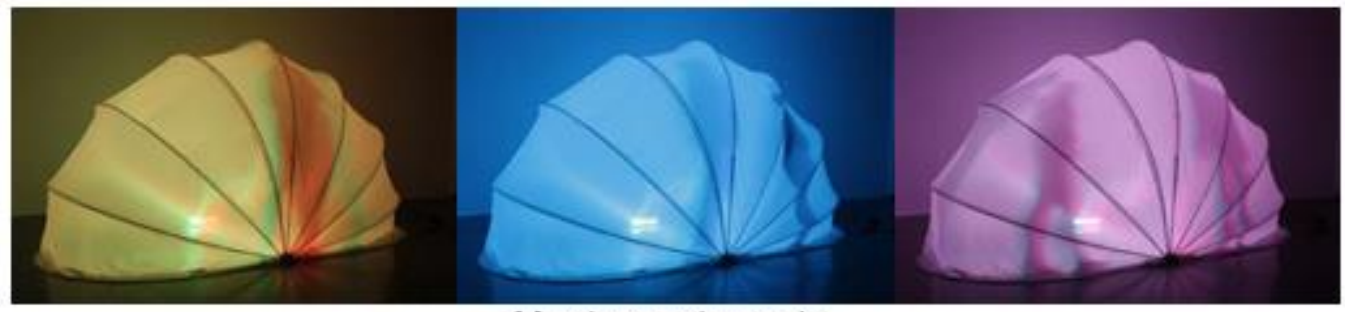

Montaje con varios usuarios.

\subsection{TECNOLOGÍA INALÁMBRICA 802.15.4}

La tecnología 802.15.4 es un estándar de comunicaciones que implementa las dos primeras capas del modelo OSI de ISO: la capa física y el control de acceso al medio. Sobre estas dos capas descansa la especificación de ZigBee aunque nosotros no vamos a utilizarla porque las características de la red que vamos a implementar no la requieren.

Mientras que otras tecnologías están diseñadas para transmitir grandes cantidades de información, nuestras necesidades se centran más en controlar un mayor número de dispositivos cuidando el consumo de los nodos para maximizar su autonomía.

Otra de las razones por los que hemos incluido esta tecnología en nuestro sistema es porque no es ampliamente utilizada por el resto de los usuarios de Processing y Arduino. Queremos entender las razones de ello y ayudar a otros usuarios a dar sus primeros pasos en la inclusión de tecnologías inalámbricas en sus proyectos. 


\subsection{SÓNAR COMO MEDIDOR DE DISTANCIAS}

La necesidad de estimar las distancias que existen entre la superficie de la carpa y la persona que se encuentre dentro desembocó en una duda en la tecnología o modo de realizar esta medición. El proceso de lluvia de ideas inicial nos aportó ideas como plagar el suelo del espacio con sensores de presión o dotar al individuo de tags RFID y localizar su posición mediante arcos detectores en cada uno de los arcos. Estas fueron quedando descartadas por su gran complejidad, aunque no descartamos retomar estas ideas para posteriores implementaciones de Leviatán.

Los sensores seleccionados fueron unos sónares para distancias, de entre 15 centímetros y 6 metros, de manera continuada y con un consumo de energía mínimo. Aunque la precisión de estos sensores no es muy alta, en esta instalación esa limitación no supuso un problema, mientras que el bajo consumo sí resultó una gran ventaja.

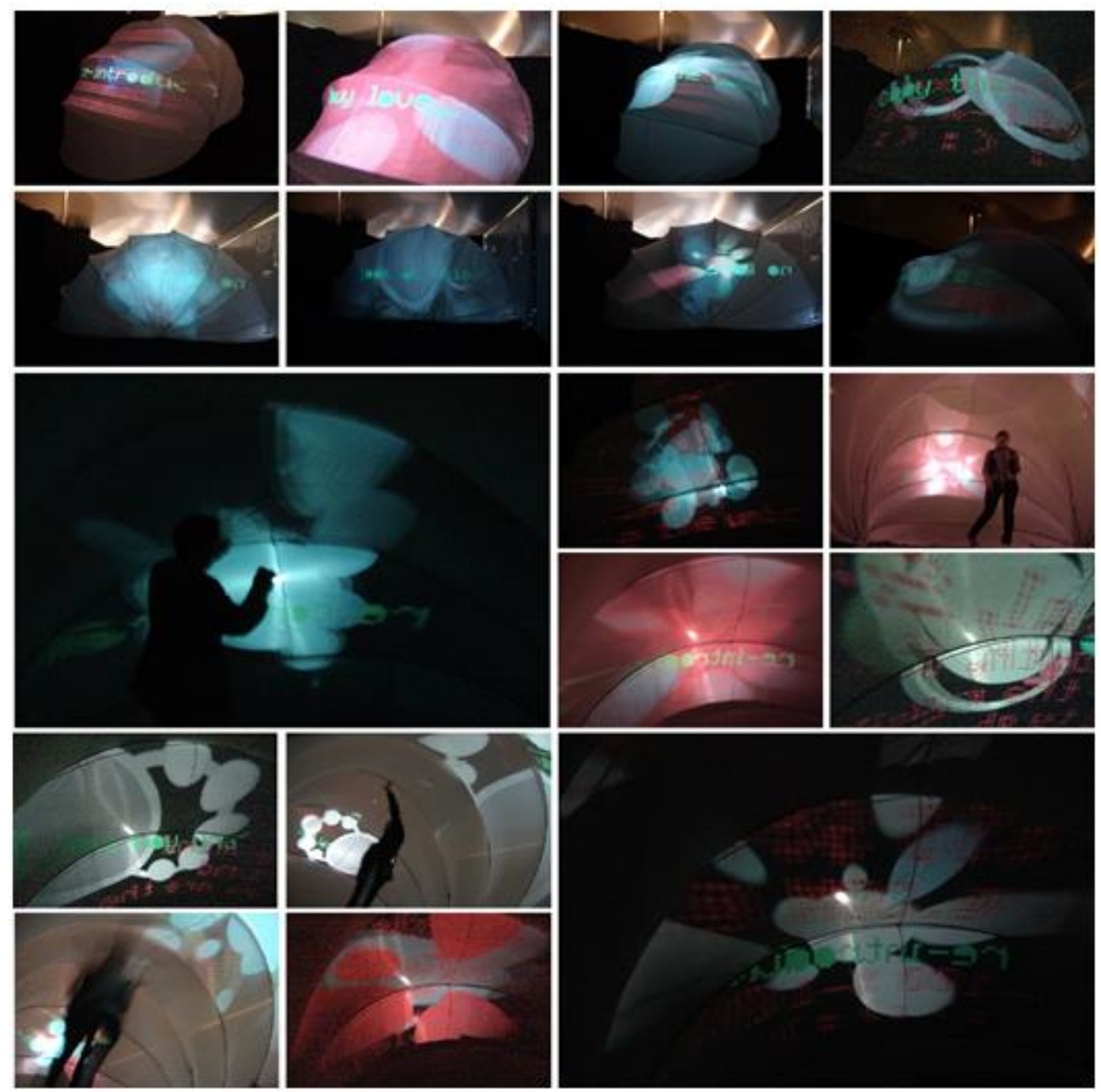

Leviatán 1.0 


\section{CONCLUSIONES}

Nuestro estudio está centrado en el desarrollo del interfaz físico desde el análisis de los modelos de interacción, y nuestros esfuerzos se orientan hacia la búsqueda o creación de una tipología de interfaces, que en un principio esbozamos y resumimos en tres, de carácter físico, de carácter multimodal (aislando la visión para reforzar los otros sentidos) y de carácter icónico.

Centramos nuestra atención en las características comunes dentro de los modelos de interacción. Analizando las estructuras de los modelos sobre los que vamos a trabajar, ya que sus aplicaciones definen la concepción del modelo de interacción y, por ende, su diseño. Estaríamos pues en un ámbito muy ampliado entre lo que sería el estudio de los modelos de interacción desde dos puntos de aplicación: uno de marcado carácter empírico sobre el que investigar y explorar en campos como la presencia, el entrenamiento, o el discernimiento social por meme cultural de los usuarios del interfaz. Otro, como ya hemos planteado, enmarcado en la investigación en producción y creación con lenguajes artísticos y performativos.

\subsection{MODELOS DE INTERACCIÓN PROPUESTOS}

La estructuración de los modelos de interacción planteados está en relación al eje singular y plural usuario/espectador, y siempre en función de las condiciones aplicadas. Para los cuales los modelos de interacción serían:

Monocanal, un solo usuario.

Multicanal, multiusuario.

Performativo, modelo combinado y abierto.

Sus aplicaciones están orientadas en dos líneas muy claramente diferenciadas, la instalación para la investigación y la instalación para el desarrollo de proyectos artísticos y/o escénicos. Para ello centramos la atención en el análisis de los modelos de interacción abiertos orientada a los contenidos con el objetivo de implementarlos sobre interfaces físicos que sean desarrollados a la vez para diferentes ámbitos y disciplina. 


\section{REFERENCIAS}

[1] Alonso Calero, J.M., Reyes-Lecuona, A., García Herrero, A. and García Berdonés, C. "Asteroide B612. Una plataforma interactiva de Realidad Virtual aplicada a una instalación de arte interactivo", Simposio Interacción 2005: pp. 251-254. Granada 2005.

[2] Gianetti, C. Estética digital, sintonía del arte, la ciencia y la tecnología, Editorial L’Angelot, Pag 119. Barcelona 2002.

[3] Igoe, T., "Making Things Talk. Practical Methods for Connecting Physical Objects", O'Reilly Media, 2007.

[4] Lozano-Hemmer, R., Catálogo “Arte Virtual: doce propuestas de arte reactivo". Ed. Chácena. Madrid 1994.

[5] McLuhan, M. Comprender los medios de comunicación. Las extensiones del ser humano. Ed. Paidos Ibérica S.A. Barcelona España 1996. Pp 43 y 44.

[6] Parés, N., Parés R. "Interaction-Driven Virtual Reality Application Design. A Particular Case: El Ball del Fanalet or Lightpools", Presence, Vol 10, No 2, pp. 236-245. Barcelona 2001.

[7] Schultz, M. "Cambios tecnológicos en el arte y en la percepción de lo humano". Revista electrónica DÓXA N². Centro de Informaciones Pedagógicas. Universidad Metropolitana de Ciencias de la Educación de Chile. p. 8. Pedagógicas. Universidad Metropolitana de Ciencias de la Educación de Chile. p. 8. Chile 2002. 